\title{
A Complete Monte Carlo and Sensitivity Analysis of Various Elements in Analog VLSI Circuits
}

\author{
Sandeep Thakur \\ Department of Electronics and Telecommunication Engineering \\ MGMCET Kamothe,India \\ Sandeepatism@gmail.com
}

\begin{abstract}
This article describes complete Monte Carlo and sensitivity analysis. Sensitivity analysis is done by various simulations which take very long time. So to save our time and to overcome complexity level of analysis. We formulate various equation in this article to observe sensitivity and MCA. We perform sensitivity analysis and Monte-Carlo Analysis $(M C A)$ on band pass filer circuit in this paper and show how the value of any component parameter change the circuit behavior. All the equation and model for sensitivity analysis of analog VLSI circuit and simulation are done with the help of MATLAB tool.
\end{abstract}

Keywords: RSS, EVA, Sensitivity, MCA, Tolerance, BPF

\section{Introduction}

An analog circuit is said to have a fault if the value of an element (component) or a measured output parameter (circuit characteristic) deviates from its designed value (including tolerances) [12]. Fault testing is performed with the objective of detection, location, or diagnosis. Fault detection simply identifies the presence of a fault in a circuit; fault location specifies the site where the circuit is faulty; fault diagnosis tells the exact value of the deviations from the nominal values.

Fault location is used for repairing faulty parts at a later time, while fault diagnosis is needed for adjustment or tuning. Such deviations are referred to as soft faults and are generally the result of tolerance violations in individual elements. Another class of faults is opens and shorts in elements and are referred to as hard faults [3-4].

An analog circuit is characterized by a number of parameters. It may be necessary to observe more than one parameter to test for faults in a circuit. This renders the testing process to be quite difficult and expensive [5-7]. Many testing algorithms have been developed but none of them offer a practical solution to the problem of fault diagnosis.

These methods use a specific type of parameter measurement or input stimuli and are therefore limited in scope. In a paper by Hamida and Kaminska [8] a method for analog circuit fault diagnosis was proposed which is based on sensitivity analysis. The method involves measuring one or more parameters and estimating sensitivity. In this paper we consider conditions for diagnosing faults in analog circuits using sensitivity analysis [9$10]$.

The design of electronic systems/devices must guarantee that all required operating and reliability parameters with minimum manufacturing costs. All types of circuits analog as well as digital, have characteristics depends upon their value of elements. These elements are non-ideal in some ways. The most important characteristics of resistor's is their resistance which is slightly different their nominal value and this characteristics also vary with the time and environment .Before designing of any circuit, the designer must know about the sensitivity of various component of circuit that may change with time and change the behavior of circuit. 
Because the circuit behavior totally depends upon their component values and if component is very critical then the circuit change its behavior with time. And we does not get expected results from our design in future. Analog VLSI circuits also having wide range of application in wireless communication. The basic part of communication device contain analog circuits.

Sensitivity analysis of analog circuit provide us the information about various component present in the circuit. The design engineer need to choose as many inexpensive components as possible, by keeping the circuit performance stable, he need to decide which elements are sensitive and how much value they required for the tolerance. With the help of sensitivity analysis we also know about the component characteristics variation in circuit and its effect on performance of system output [11].

All circuits, analog or digital, have characteristics dependent on the values of their component parts. Invariably, these parts are non-ideal in many ways. Even their most fundamental characteristics, for example a resistor's resistance, will be at least slightly different from the specified value. These characteristics also can vary over time and environmental conditions.

The wise circuit designer learns how to analyze the sensitivities of circuits' critical characteristics to the variations in the components. With this knowledge, the designer can better decide how to balance any tradeoffs between performance and cost. Deeper use of sensitivity analysis can lead designers to modify circuit topologies, or make completely different choices in order to optimize these tradeoffs.

This paper organized in five sections. First section deals with the introduction. Second section describe the sensitivity and its general equation. Third section deals with result and discussion. This section contain various simulation of sensitivity performed for band pass filter. Fourth section describe the result analysis and last section deals with conclusion of whole work done in this paper.

\section{Theory of Sensitivity}

A simple definition of sensitivity is how much specific system behavior/characteristic changes as a individual component value changes [12-13]. The general equation for sensitivity analysis is given below

$S_{x}^{y}=\lim _{\Delta x \rightarrow 0} \frac{\frac{\Delta y}{y}}{\frac{\Delta x}{x}}=\frac{x}{y} \frac{\partial y}{\partial x}$

Equation (1) is the general mathematical definition of circuit sensitivity: Where $S$ represent sensitivity, $\mathrm{X}$ represent changing element/component and $\mathrm{Y}$ is the characteristic of circuit which one want to evaluate as component value is varied[14-15].

The middle part of this equation shows that the percentage that the dependent variable $\Delta y / y$ changes, relative to the percentage that the independent variable $\Delta x / x$ changes. The sensitivity analysis done by using these formulae derived below. Let's take a transfer function $\mathrm{H}(\mathrm{s})$.

$\mathrm{H}(s)=\frac{N(s)}{D(s)}$

Here $\mathrm{N}(\mathrm{s})$ represent the numerator part of transfer function and $\mathrm{D}(\mathrm{s})$ represent the denominator part of transfer function. From equation (1) and equation (2), we write a new equation which is same as equation (1). But its variable name are changed to make our calculation easy. In general, the AC-sensitivity is given by the following equation:

$\operatorname{Sens}(H(s), W)=\frac{W}{H(s)} \frac{\partial H(s)}{\partial W}$

Substituting equation (1) into (2) and applying the chain rule have 
$\operatorname{Sens}(H(s), W)=W\left(\frac{1}{N(s)} \frac{\partial N(s)}{\partial W}\right)-\left(\frac{1}{D(s)} \frac{\partial D(s)}{\partial W}\right)$

Here $\mathrm{W}$ is the component which one want to vary w.r.t. circuit transfer function. By using above equation (3) we can calculate the sensitivity of circuit any circuit.

\subsection{Sensitivity and Monotonicity Analysis of Band Pass Filter}

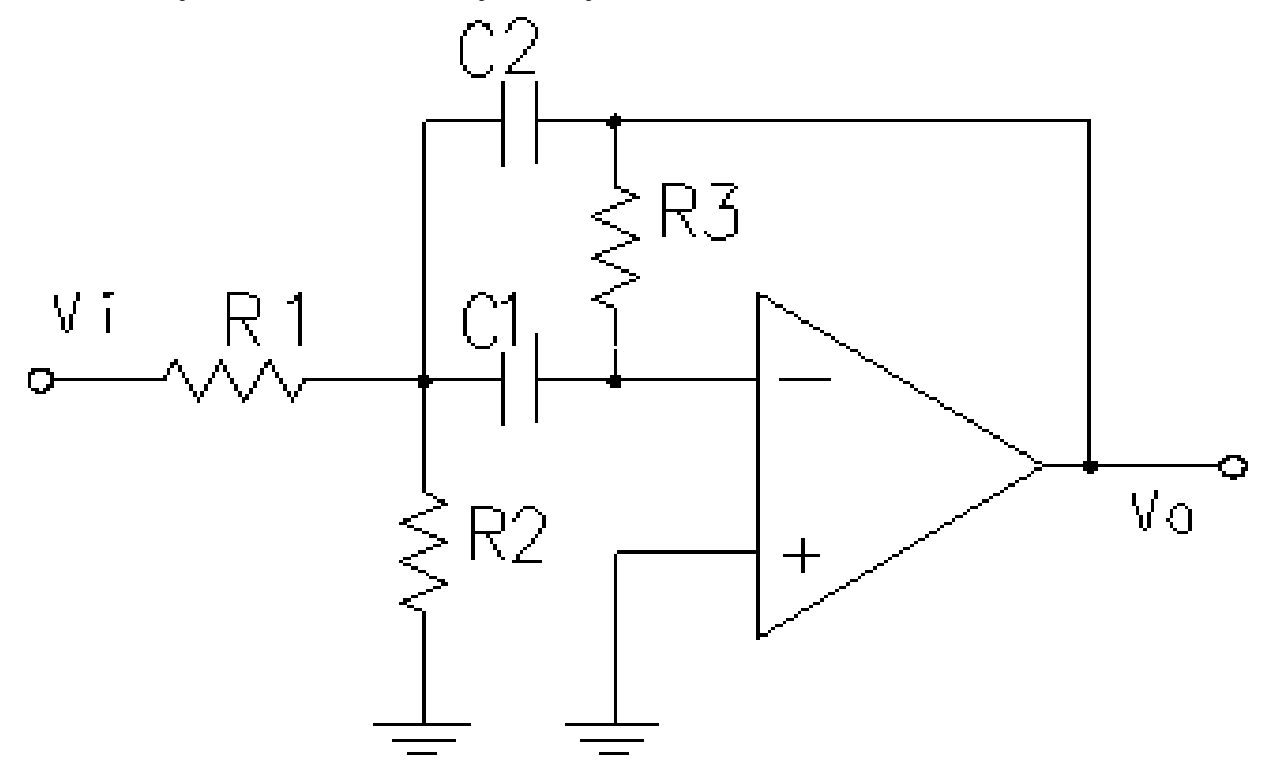

Figure 1. Circuit Diagram of BPF

The filter has a centre frequency of $\mathrm{f} 0=500 \mathrm{~Hz}$ and $\mathrm{Q}=20$. The component values are $\mathrm{R} 1=6.34 \mathrm{~K}, \mathrm{R} 2=80.63, \mathrm{R} 3=127 \mathrm{~K}$. The values of capacitor are $\mathrm{C} 1=\mathrm{C} 2=0.1 \mathrm{uF}$. By using equation 3 we drive sensitivities expression for band pass filter [16-21].

Here $\mathrm{wo}=2 \pi \mathrm{fo}$ and $\mathrm{G}=$ Design gain

The equation for sensitivity of $\mathrm{R} 1$ resistor is given below

$S_{R 1}(m)=-1$ (Constant)

Similarly sensitivity of $\mathrm{R} 2$ is

$S_{R 2}(m)=\frac{w o^{2}\left(2 Q^{2}-G\right)\left(w o^{2}-m^{2}\right)}{2\left[Q^{2}\left(\left(w o^{2}-m^{2}\right)+(m \cdot w o)^{2}\right]\right.}$

Similarly sensitivity of R3 is

$S_{R 3}(m)=\frac{w o^{2}\left[Q^{2}\left(w o^{2}-m^{2}\right)+m^{2}\right]}{Q^{2}\left(\left(w o^{2}-m^{2}\right)+(m \cdot w o)^{2}\right]}$

Similarly sensitivity of $\mathrm{C} 1$ is

$S_{C 1}(m)=\frac{m^{2}\left[\left(2 Q^{2}\right)\left(w o^{2}-m^{2}\right)+w o^{2}\right.}{2\left[Q^{2}\left(\left(w o^{2}-m^{2}\right)^{2}+(m \cdot w o)^{2}\right]\right.}$

Similarly sensitivity of $\mathrm{C} 2$ is

$S_{C 2}(m)=\frac{m^{2}\left[\left(2 Q^{2}\right)\left(w o^{2}-m^{2}\right)-w o^{2}\right.}{2\left[Q^{2}\left(\left(w o^{2}-m^{2}\right)^{2}+(m \cdot w o)^{2}\right]\right.}$

The equation (4), (5), (6), (7) and (8) describe sensitivities of all component of band pass filer. By using those equations we can plot sensitivities of BPF. 


\section{Sensitivity and Monte Carlo analysis of BPF}

\subsection{Sensitivity of BPF}

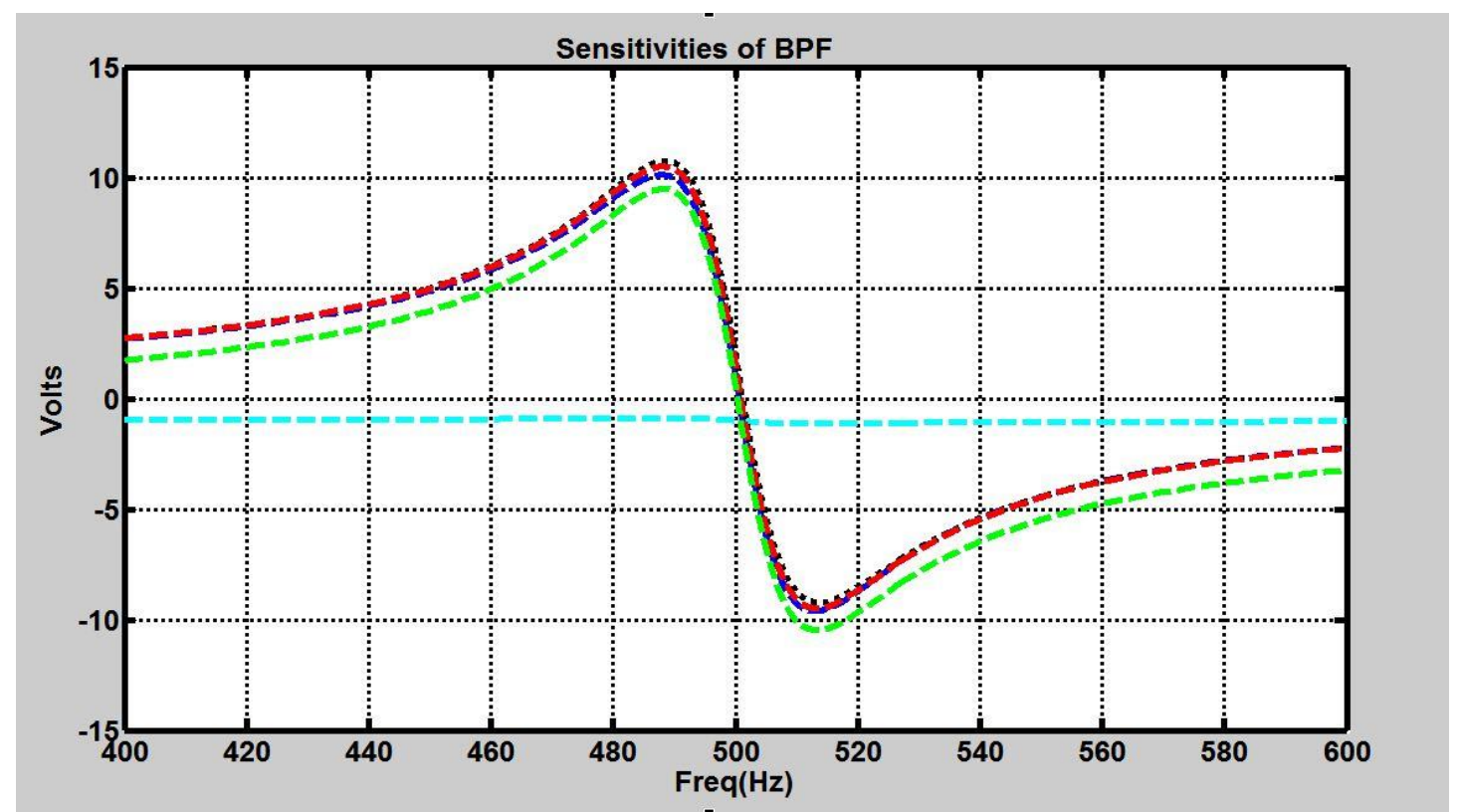

Figure 2. Sensitivities of R1, R2, R3, C1 and C2

Here we can see that the sensitivity of R1 is exactly -1 in Figure 2 . We can see that the sensitivities of R2, R3, C1 and C2 are almost overlays.

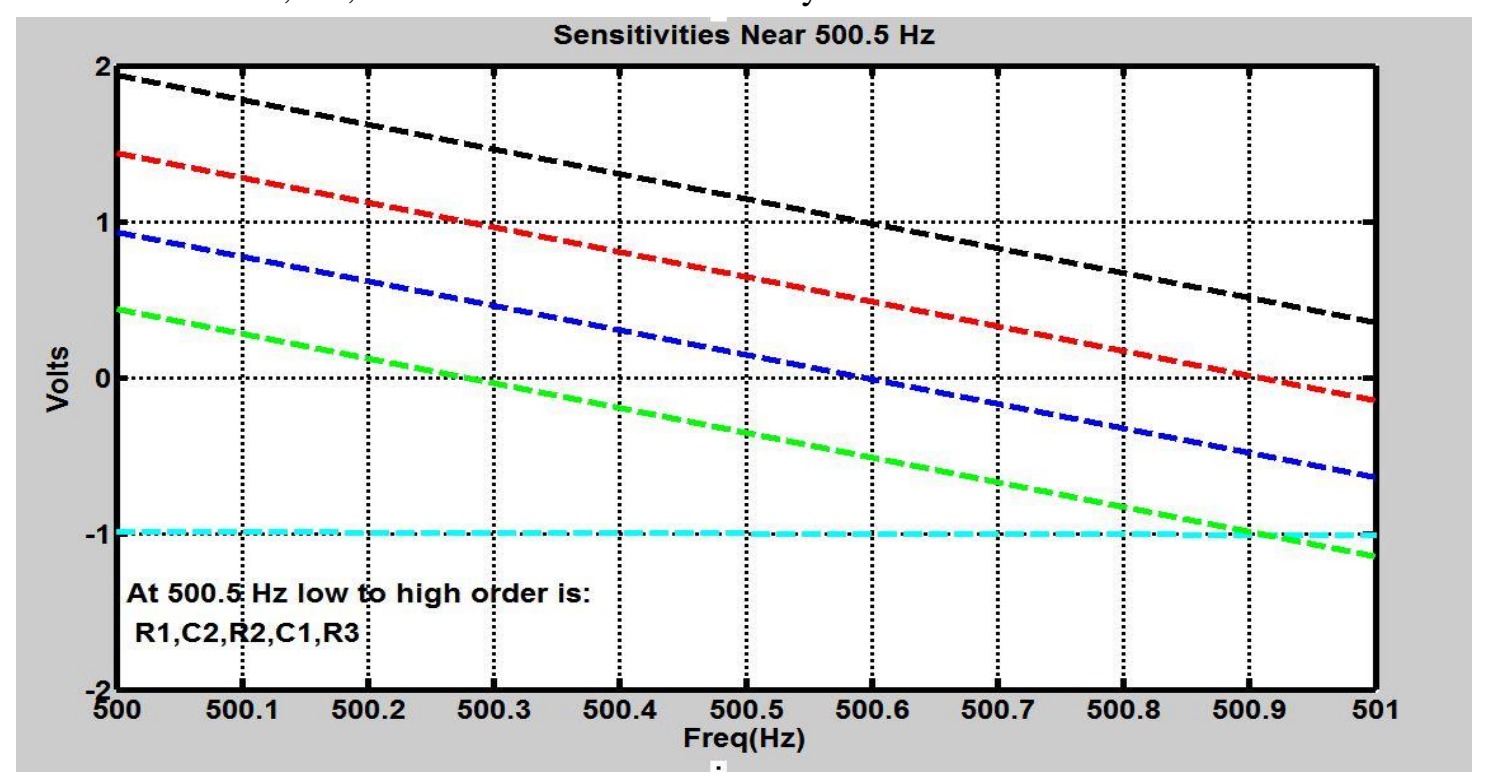

Figure 3. Sensitivities of R1, R2, R3, C1 and C2 Near $500.5 \mathrm{~Hz}$

Figure 3, shows the sensitivities of R1, R2, R3, C1 and C2 near $500.5 \mathrm{~Hz}$ frequency. The sensitivity of R1 is constant and it is calculated in equation (4). The green dotted line shows sensitivity of capacitor $\mathrm{C} 2$ which is calculated in equation (8). The blue dotted line shows sensitivity of capacitor R2 which is calculated in equation (5). The red dotted line shows sensitivity of capacitor $\mathrm{C} 1$ which is calculated in equation (7). The black dotted line shows sensitivity of capacitor $\mathrm{C} 1$ which is calculated in equation (7). 


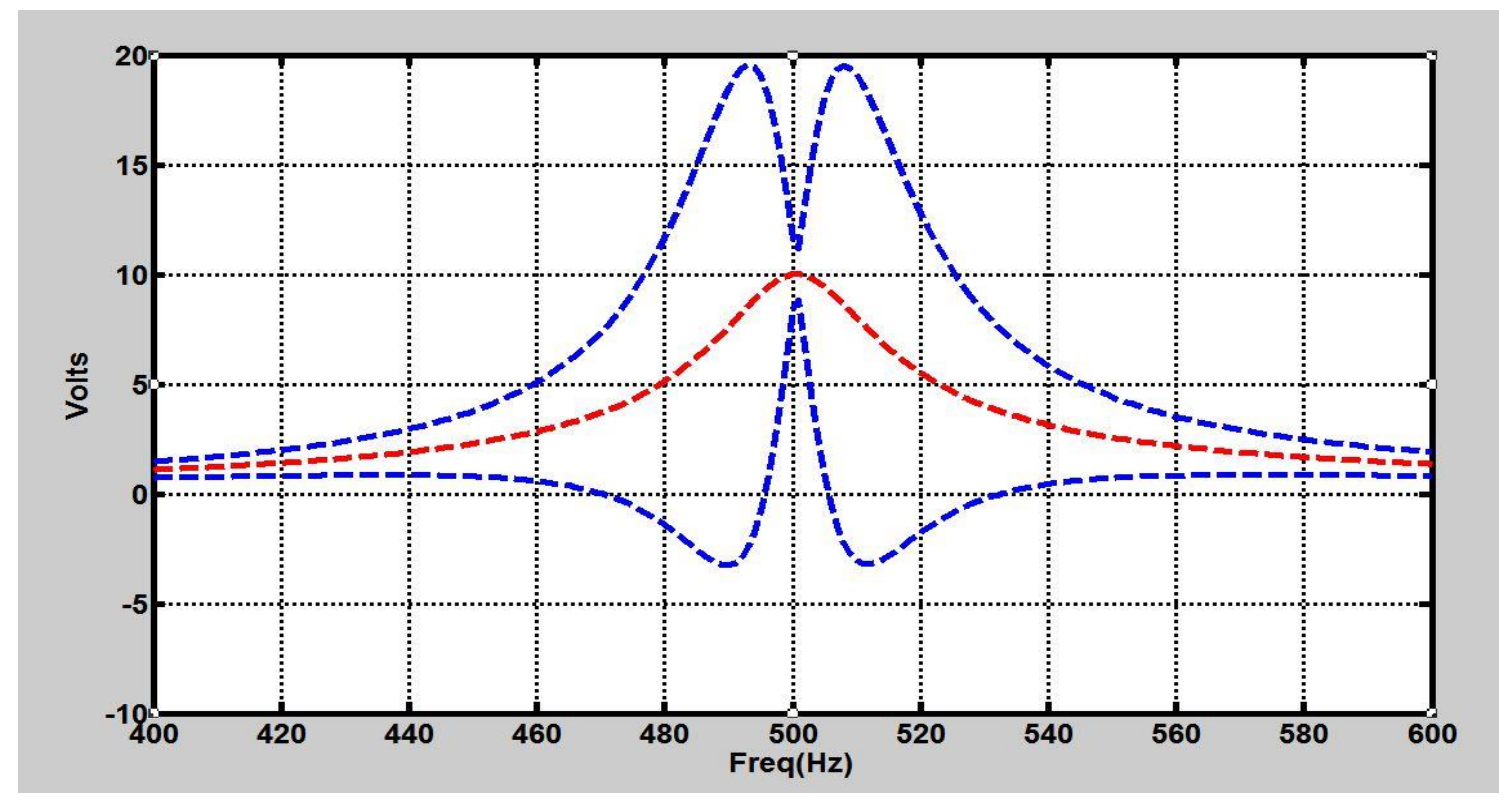

Figure 4. Root Sum Square Analysis

The nominal output Vo in Figure 4, can be seen peaking at $10 \mathrm{~V}$ at $500 \mathrm{~Hz}$ with $\mathrm{Q}=20$ as designed.

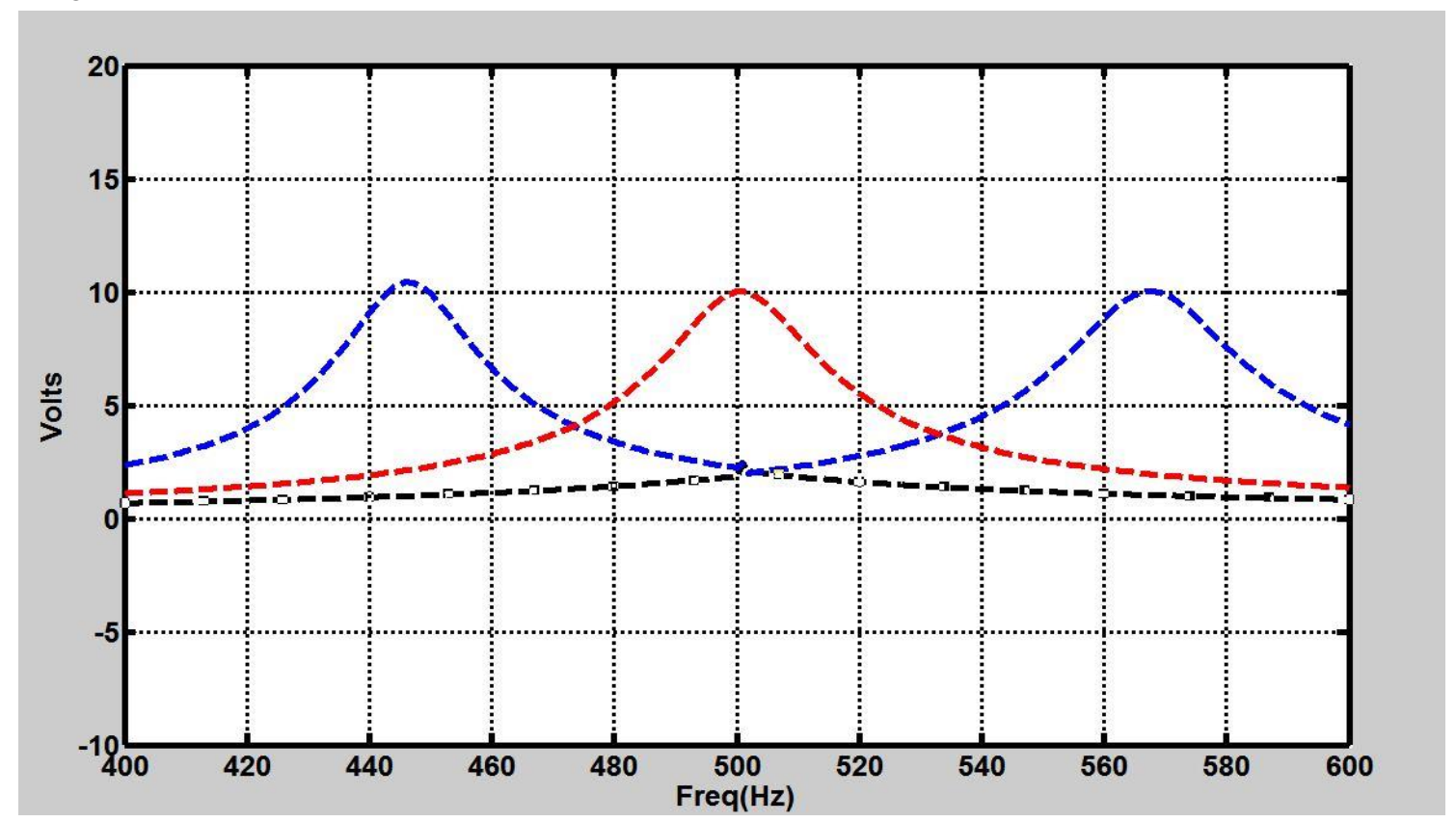

Figure 5. Extreme Value Analysis

The extreme value plot in Figure 5, shows that low centre frequency peak is $447 \mathrm{Hzwhile} \mathrm{high} \mathrm{is} \mathrm{at} 568 \mathrm{~Hz}$

\subsection{Monte Carlo Analysis of BPF}

The general Monte Carlo analysis procedure is to create random number for each of $\mathrm{M}$ component then convert these random number to $M$ tolerance multiplier. If a decimal 
tolerance $\operatorname{Tr}$ is given a random tolerance multiplier between $1-\operatorname{Tr}$ and $1+\operatorname{Tr}$ is calculated. These are applied to $\mathrm{M}$ component in circuits [22-24].

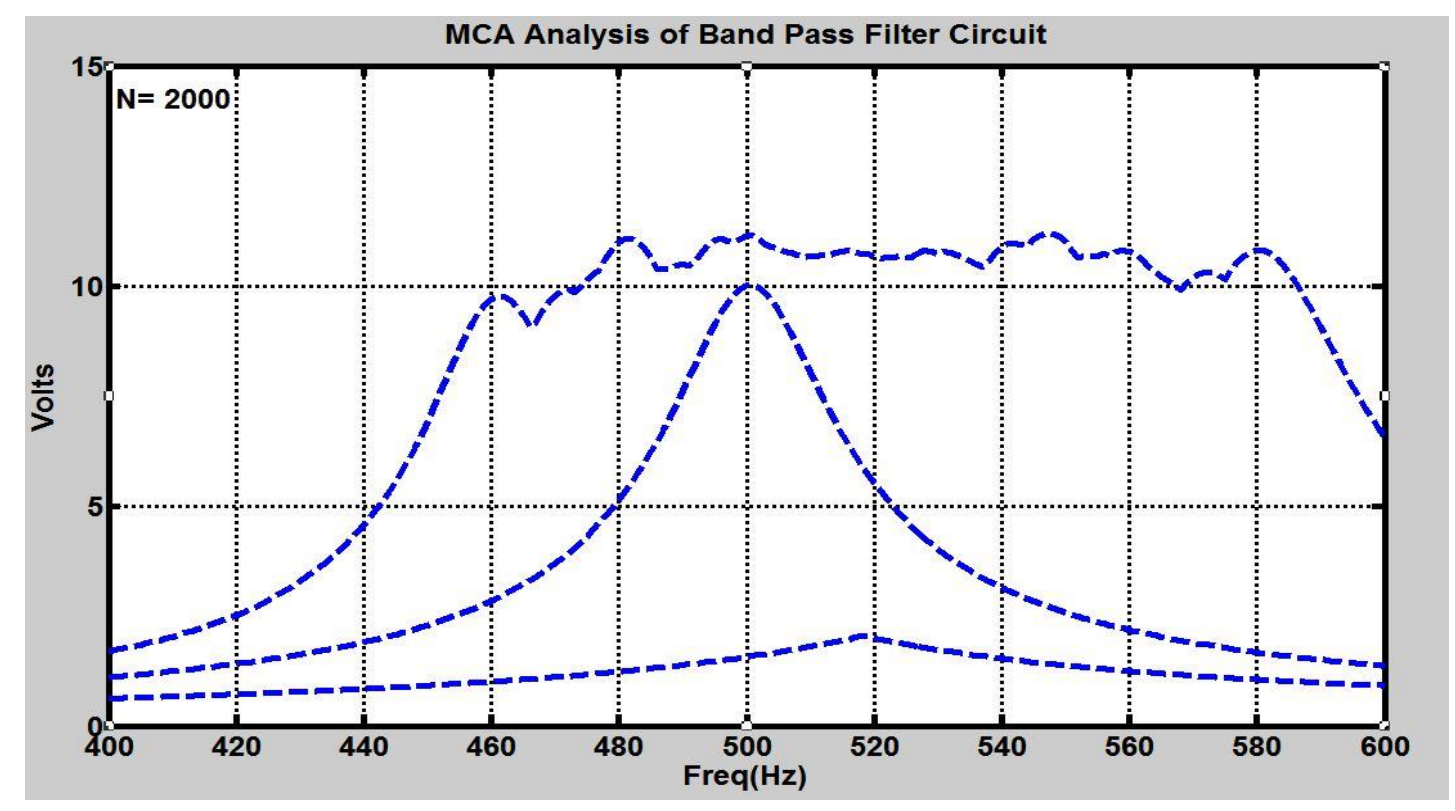

Figure 6. Monte Carlo Analysis of BPF (Uniform Distribution)

In Figure 6, both the left and right centre frequency have increased considerably. In Figure 6, shows MCA for symmetric tolerance.

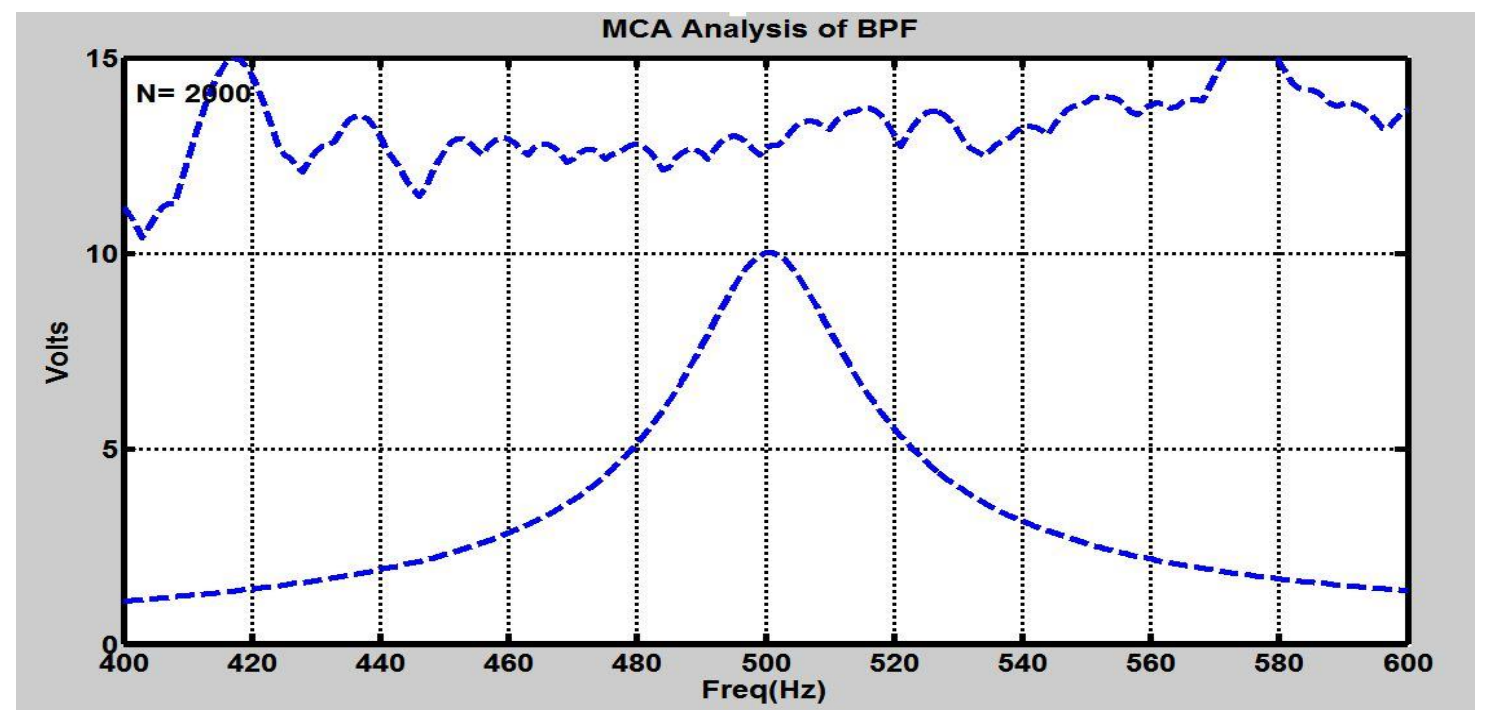

Figure 7. Monte Carlo Analysis of BPF (Normal Distribution)

In Figure 7, shows MCA for band pass filter when N=2000. 


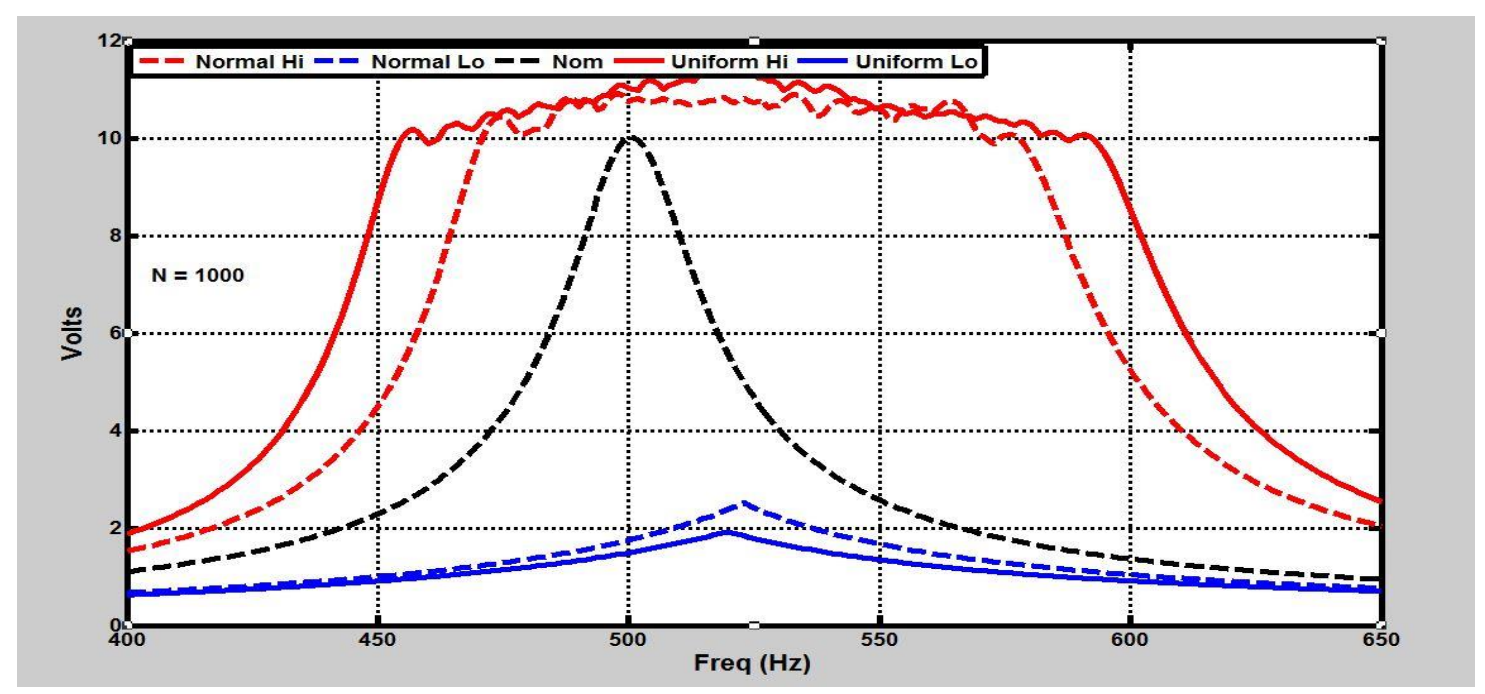

Figure 8. Monte Carlo Analysis of BPF at $\mathrm{N}=\mathbf{1 0 0 0}$

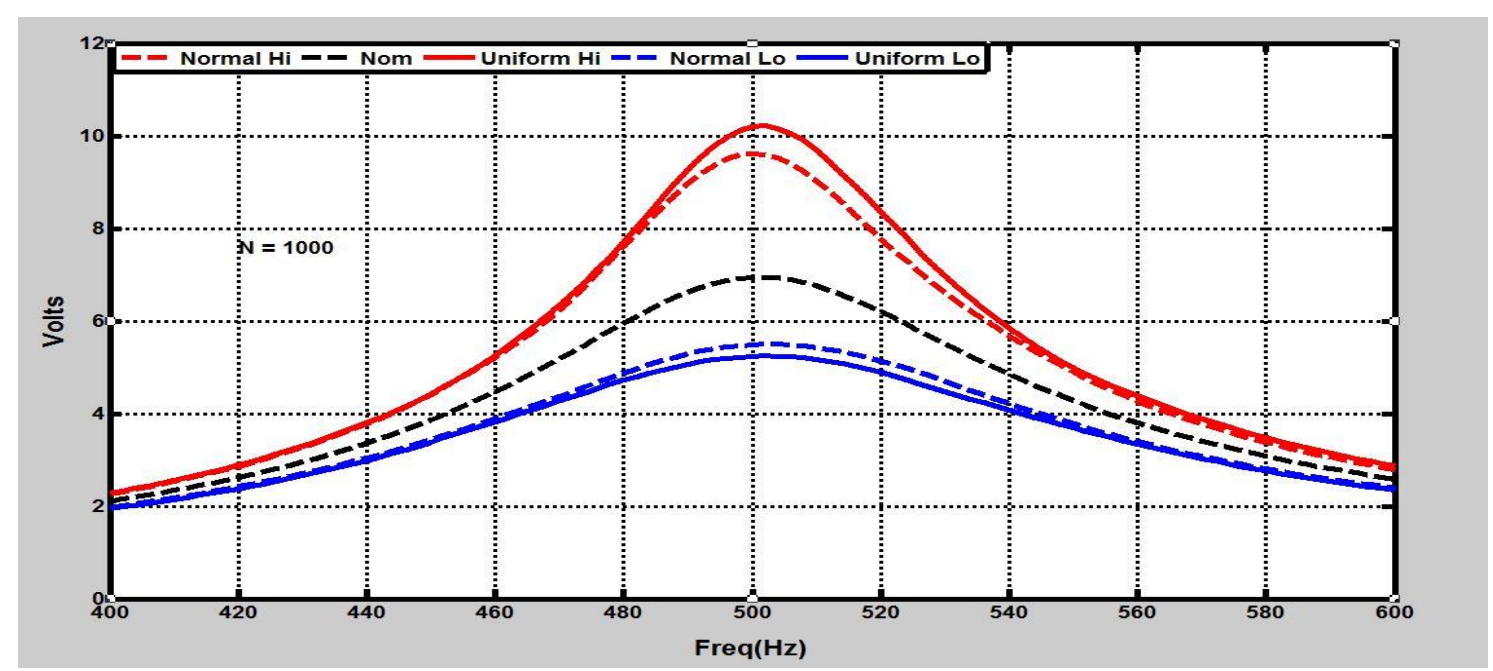

Figure 9. Monte Carlo Analysis of Sallen key filter at $\mathrm{N}=1000$

In Figure 8, shows MCA for band pass filter when N=1000. Figure 9, shows MCA for sallen key filter at $\mathrm{N}=1000$ for various distribution.

\subsection{Sensitivity VS Monotonicity analysis BPF}

The Figure 10, below shows a $-20 \%$ to $+20 \%$ (factor of 0.8 to 1.2 ) variation of the capacitance value of $\mathrm{C} 1$. The rightmost curve is at a fixed frequency of $470 \mathrm{~Hz}$, while the one to the left of it is at a fixed frequency of $480 \mathrm{~Hz}$. One can easily see that the degree of non-monotonicity is somewhat severe. 


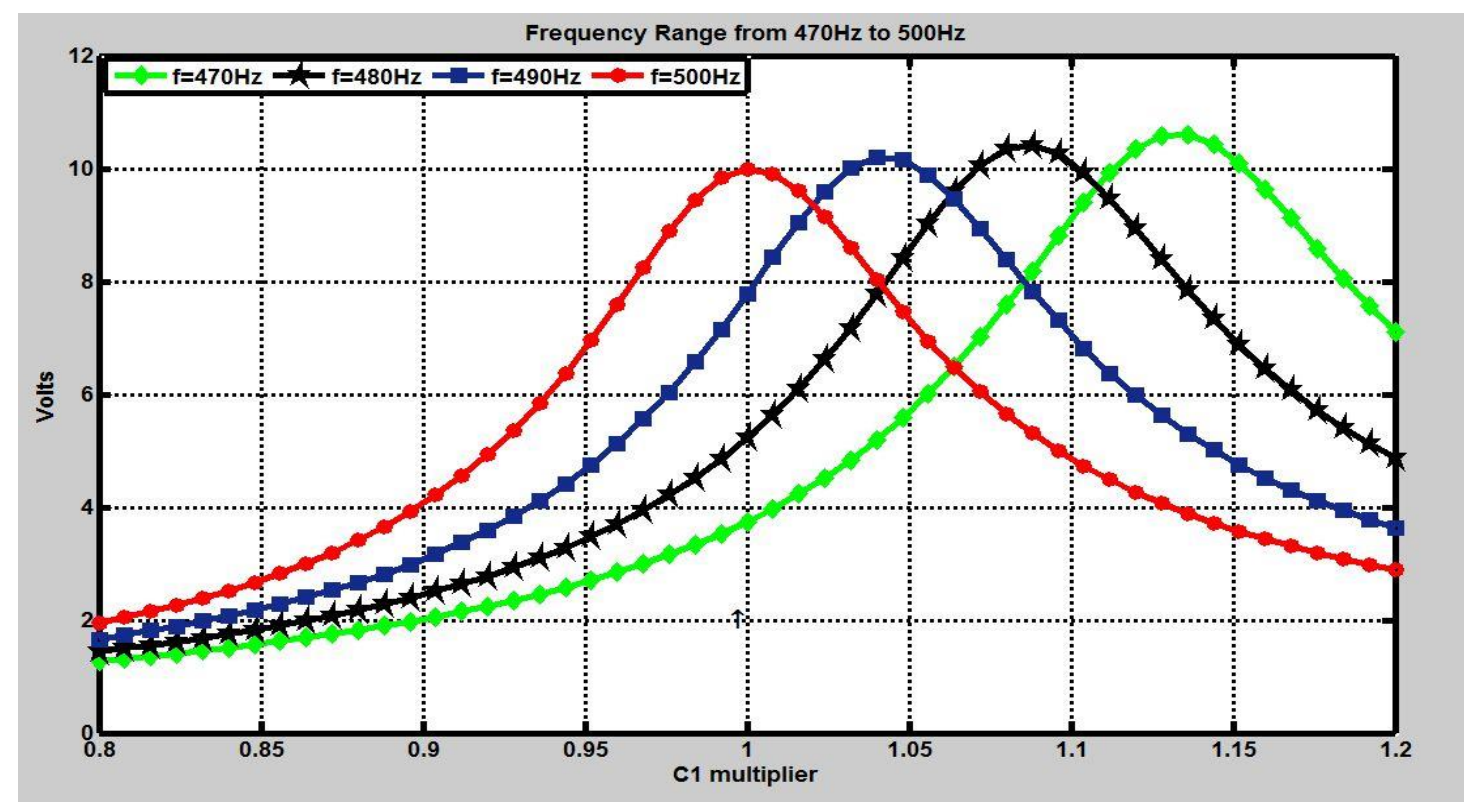

Figure 10. Sensitivity of $\mathrm{C} 1$ in Band Pass Filter

By calculating the slopes at the nominal value of $\mathrm{C} 1$ we can plot the normalized sensitivity of $\mathrm{C} 1$ vs. frequency on the second plot below Figure 11.

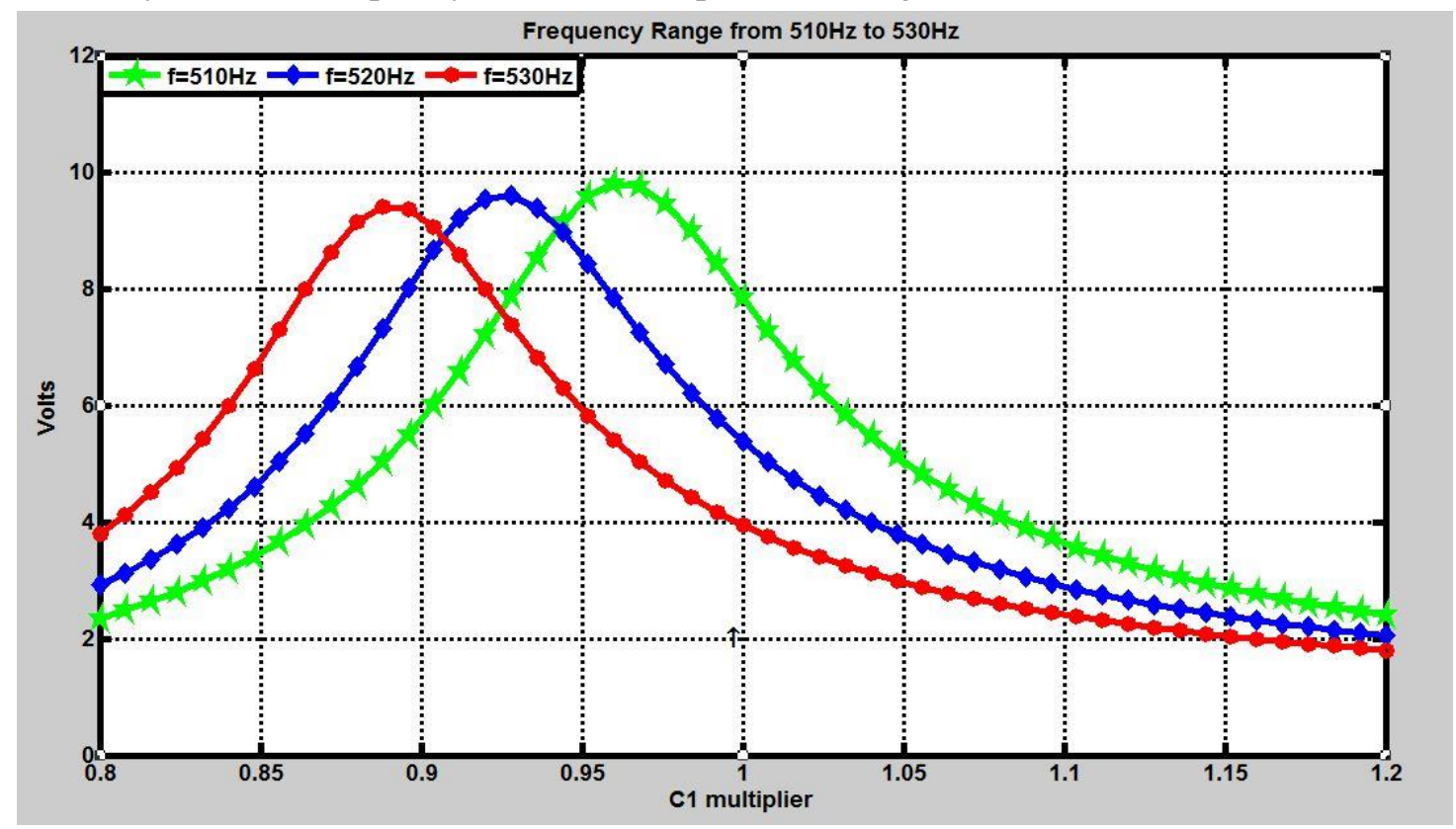

Figure 11. Sensitivity of $\mathrm{C} 1$ in Band Pass Filter at $510 \mathrm{~Hz}$ to $530 \mathrm{~Hz}$ 


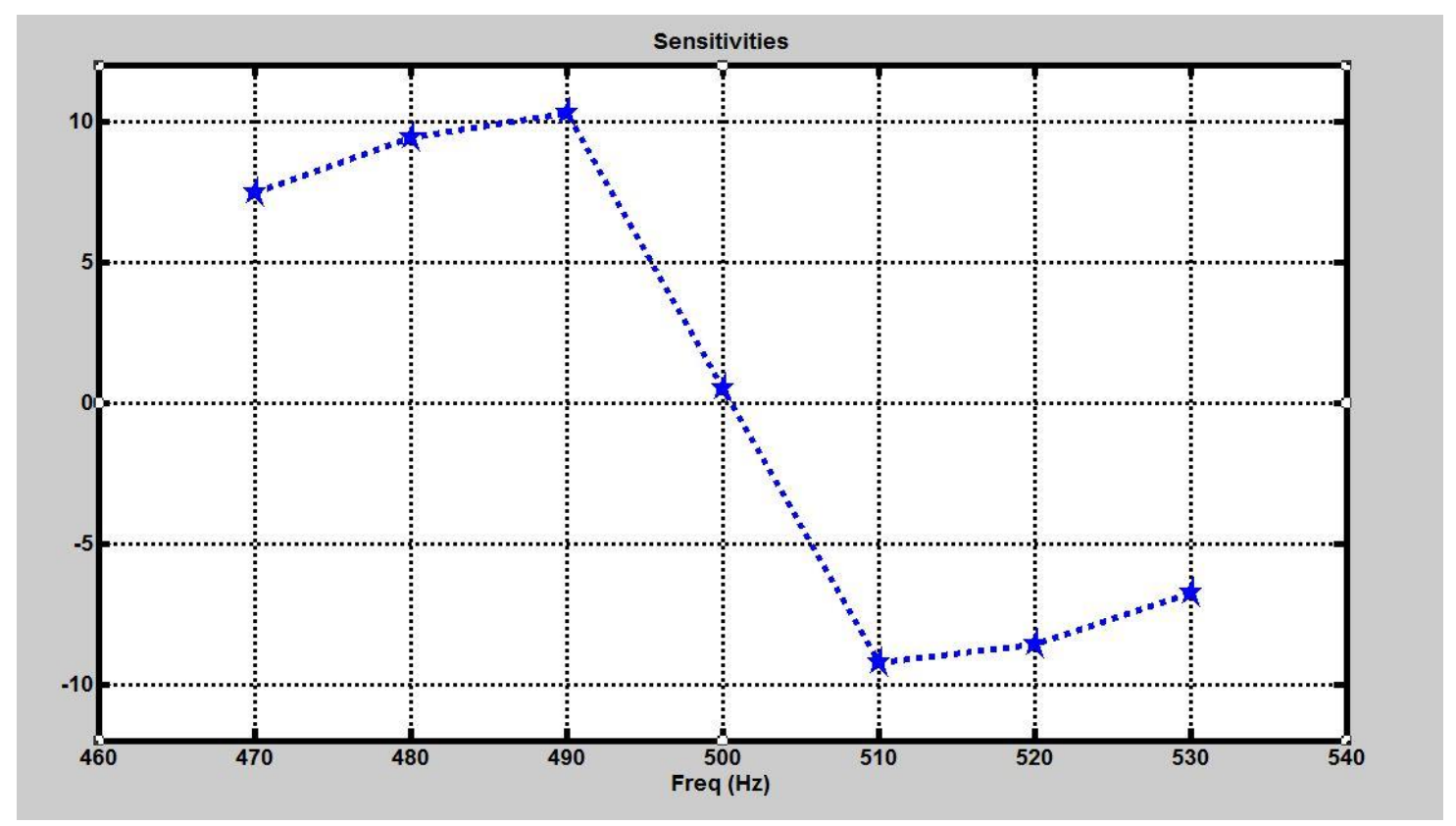

Figure 12. Normalized Slope

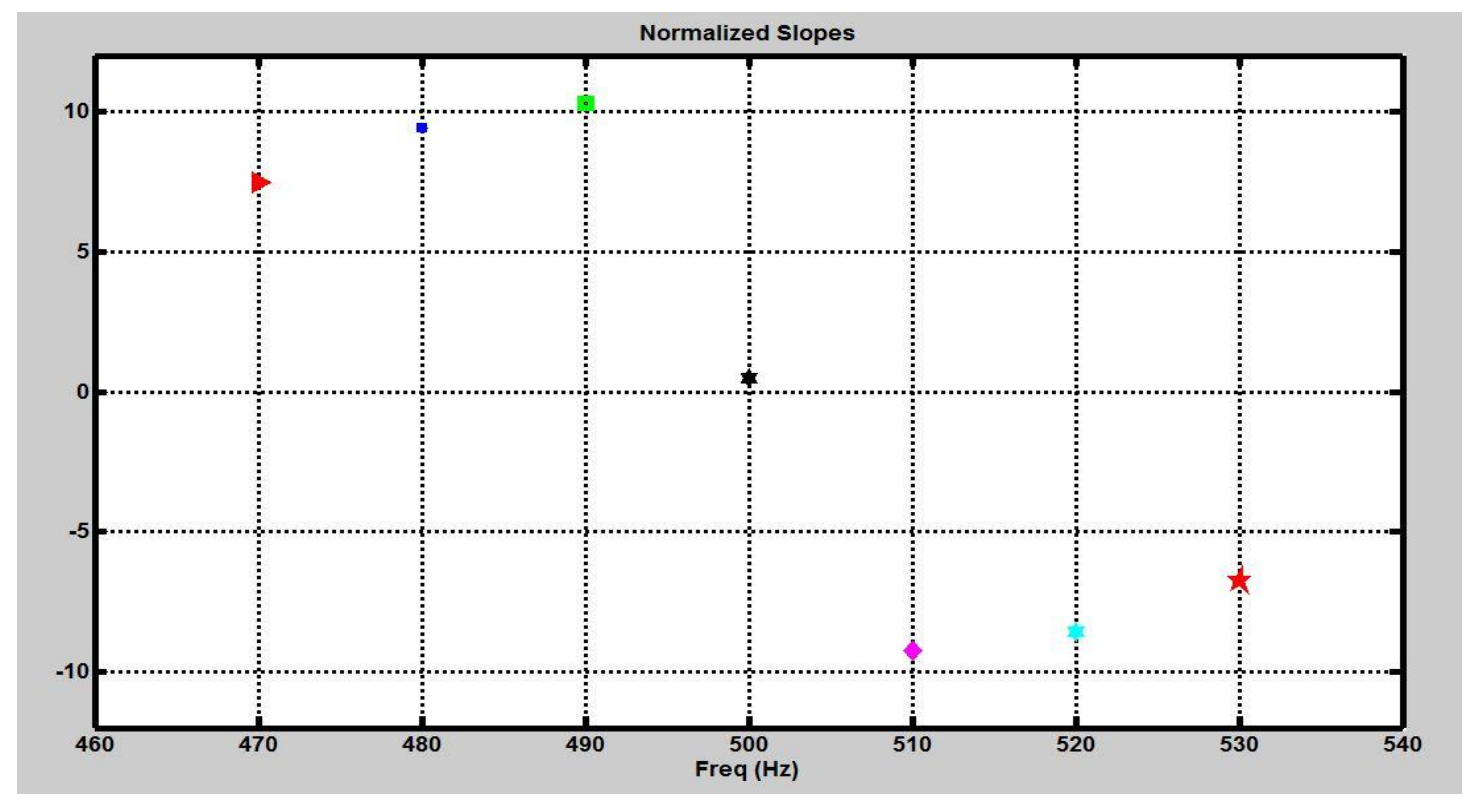

Figure 13. Normalized Slope at Particular Points

(Recall that the normalized sensitivity is the slope $\mathrm{dVo} / \mathrm{dC} 1$ multiplied by $\mathrm{C} 1 / \mathrm{Vo}$; in effect, a normalized slope.) Hence the normalized sensitivity of $470 \mathrm{~Hz}$ is about $6 \%$, while the $480 \mathrm{~Hz}$ point is about $8 \%$.

\section{Result Analysis}

By performing this analysis, we learn various aspects of analog VLSI circuits. That is calculation of $\mathrm{Q}$ sensitivities are very easy task. This analysis also provide us a valuable information of component sensitivity, by using that we can set the tolerance of component in the circuit. For example, if the component is very sensitive then we have to specify the tolerance of that component very close to nominal value. 
This analysis reduce the designing cost of analog VLSI circuits and also provide the information about tolerance of component, which made our design cheaper and give expected results. Due to this we also increase the aging factor of the device.

Sensitivity analysis also used to test the various faults of analog circuit, which occur during the manufacturing process. So it is very important method to analyze any circuit before implementation in system. In this article we analyzed various component of band pass filter using sensitivity and Monte Carlo analysis with the help of MATLAB tool.

\section{Conclusion}

In this paper we analyses the Monte Carlo and sensitivity analysis of band pass filter circuit. This analysis is very helpful for designer to choose particular value of component, so that the output of system should be stable and does not change the characteristics of output. This paper contain simulation results for Monte Carlo and sensitivity analysis using MATLAB software. The equation we drive in this article are plotted with the help of simulation software.

\section{References}

[1] Y. Lu and R. Dandapani," Hard Fault Diagnosis in Analog Circuits Using Sensitivity Analysis”, Proc. of the IEEE VLSI Test Symp., (1993) April, pp. 225-229.

[2] V. dePlassche and J. Rudy, "CMOS integrated analog-to-digital and digital-to-analog converters", Springer Science \& Business Media, vol. 742, (2013).

[3] S. Thakur and B. Raj, "Fault Modeling and Parametric Fault Detection in Analog VLSI Circuits using Discretization", (2016).

[4] L. Milor and A. L. Sangiovanni-Vincentelli, "Minimizing ProductionTest Time to Detect Faults in Analog Circuits," IEEETrans. on Computer-Aided Design, vol. 13, (1994) June, pp. 796-813.

[5] K. Maggard, P. Karunaratna and C. Stroud, "Built-InSelf Test for analog circuits in Mixed Signal System”, Proc.IEEE Southeast Regional Conf., (1999).

[6] J. Cui and Y. Wang, "A novel approach of analog circuit fault diagnosis using support vector machines classifier", Measurement, vol. 44, no. 1, (2011), pp. 281-289.

[7] S. Thakur and A. Naithani, "Touch Mode Analysis and Simulation of Capacitive Pressure Sensor Based on MEMS", (2016).

[8] N. B. Hamida and B. Kaminska, "Multiple Fault Analog Circuit Testing by Sensitivity Analysis," Journal Electronic Testing: Theory and Applications, vol. 4, (1993) November, pp. 331-343.

[9] S. Thakur, "Modeling and Performance Analysis of MEMS Based Capacitive Pressure Sensor Using Different Thickness of Diaphragm", (2016).

[10] A. Osseiran (Ed.), "Analog and mixed-signal boundary-scan", A guide to the IEEE 1149.4 test standard. Springer Science \& Business Media, vol. 16. (2013).

[11] S.Thakur, "A Comprehensive Approach for Modeling and Diagnosis of Various Faults in Analog VLSI Circuits", (2016).

[12] H. Luo, Y. Wang and J. Cui, "A SVDD approach of fuzzy classification for analog circuit fault diagnosis with FWT as preprocessor", Expert Systems with Applications, vol. 38, no. 8, (2011), pp. 10554-10561.

[13] S. Thakur and K. V. V. Satyanarayana, "Analytical modeling and sensitivity analysis of clamped edge circular diaphragm based on capacitive pressure sensing method", In Intelligent Systems and Control (ISCO), 2016 10th International Conference on, IEEE, (2016), pp. 1-5.

[14] N. Balabanian, T. A. Bickart and S. Seshu, "Electrical Network Theory", John Wiley \& Sons, Inc., (1969).

[15] L. Yuan, Y. He, J. Huang and Y. Sun, “A new neural-network-based fault diagnosis approach for analog circuits by using kurtosis and entropy as a preprocessor", IEEE Transactions on Instrumentation and Measurement, vol. 59, no. 3, (2010), pp. 586-595.

[16] S. Thakur and B. Raj, "Tolerance Analysis of Analog VLSI Circuits using Sensitivity", (2016).

[17] C Yang, S. Tian, B. Long and F. Chen, "Methods of handling the tolerance and test-point selection problem for analog-circuit fault diagnosis", IEEE Transactions on Instrumentation and Measurement, vol. 60, no. 1, (2011), pp. 176-185.

[18] T. C. Lin, "'Analog circuit fault diagnosis under parameter variations based on type-2 fuzzy logic systems", International Journal of Innovative Computing, Information and Control, vol. 6, no. 5, (2010), pp. 2137-2158.

[19] J. Vineela, G. Praneetha, R. Harshad, K. Masrunnisa, M. Pruthvi Kumar and T. Sandeep, "A Complete Analysis of Tolerance of Component in Analog VLSI Circuits Using Sensitivity", International Journal of Hybrid Information Technology, vol. 9, no. 7, (2016), pp. 9-18. 
[20] A. S. S. Vasan, B. Long and M. Pecht, "Diagnostics and prognostics method for analog electronic circuits", IEEE Transactions on Industrial Electronics, vol. 60, no. 11, (2013), pp. 5277-5291.

[21] A. C. S. Borbon and E. T. Cuautle, "Symbolic sensitivity analysis in the sizing of analog integrated circuits", Computing Science and Automatic Control, (2013).

[22] S. Thakur, K. V. V. Satyanarayana and K. C. M. Reddy, "Diagnosis of parametric faults in linear analog VLSI circuits", In Intelligent Systems and Control (ISCO), 2016 10th International Conference on, IEEE, (2016), pp. 1-5..

[23] L. P. Huelsman and P. E. Allen, "Introduction to the Theory and Design of Active Filters", McGrawHill, New York, (1980).

[24] S. Thakur and N. Kumar, "Design and Analysis of Multi Input Logic Gates Based on Quantum Dot Cellular Automata", IJRECE, vol. 3, no. 3, (2015), pp. 70-73. 
International Journal of $\mathrm{u}-$ and $\mathrm{e}-$ Service, Science and Technology Vol. 9, No. 11 (2016) 